\title{
Efficacy and safety of Chinese herbal medicine for benign prostatic hyperplasia: systematic review of randomized controlled trials
}

\author{
Chun Ho Ma ${ }^{1}$, Wai Ling Lin $^{1}$, Sing Leung Lui ${ }^{2}$, Xun-Yuan Cai ${ }^{1}$, Vivian Taam Wong ${ }^{3}$ Eric Ziea ${ }^{3}$ \\ and Zhang-Jin Zhang ${ }^{1}$
}

\begin{abstract}
Chinese herbal medicine is commonly used as a treatment for benign prostatic hyperplasia (BPH), but its efficacy and safety remain to be examined. To compare the efficacy and adverse events of Chinese herbal medicine alone or used adjuvantly with Western medications for BPH. Two independent reviewers searched the major electronic databases for randomized controlled trials comparing Chinese herbal medicine, either in single or adjuvant use with Western medication, with placebo or Western medication. Relevant journals and grey literature were also hand-searched. The outcome measures included changes in urological symptoms, urodynamic measures, prostate volume and adverse events. The frequency of commonly used herbs was also identified. Out of 13922 identified citations of publications, 31 studies were included. Eleven studies with a Jadad score $\geqslant 3$ were selected for meta-analysis. Chinese herbal medicine was superior to Western medication in improving quality of life and reducing prostate volume. The frequency of adverse events in Chinese herbal medicine was similar to that of placebo and less than that of Western medication. The evidence is too weak to support the efficacy of Chinese herbal medicine for BPH due to the poor methodological quality and small number of trials included. The commonly used herbs identified here should provide insights for future clinical practice and research. Larger randomized controlled trials of better quality are needed to truly evaluate the efficacy of Chinese herbal medicine.
\end{abstract}

Asian Journal of Andrology (2013) 15, 471-482; doi:10.1038/aja.2012.173; published online 3 June 2013

Keywords: benign prostatic hyperplasia (BPH); meta-analysis; review; traditional Chinese medicine

\section{INTRODUCTION}

Benign prostatic hyperplasia (BPH) is a pathological condition involving the cellular proliferation of the epithelial and stromal cells in the prostate gland. ${ }^{1}$ BPH clinically manifests itself with lower urinary tract symptoms (LUTS) that include urinary intermittency, frequency, straining, urgency, weak stream, incomplete emptying and nocturia. ${ }^{2}$ LUTS has a significant negative impact on quality of life (QoL) and causes a great number of middle-aged to elderly men to seek treatment. ${ }^{3}$ In the United States, the prevalence of Caucasian men aged 50-79 years who were expected to meet the criteria for seeking treatment options for BPH was 6.5 out of 27 million (24\%) in 2000. ${ }^{4} \mathrm{BPH}$ is not only prevalent in the United State $^{5}$ and Europe, ${ }^{6-11}$ but is becoming increasingly common in Asian, ${ }^{12}$ including Japan, ${ }^{13}$ Korea, ${ }^{14}$ Mainland China ${ }^{15}$ and Hong Kong region. ${ }^{16} \mathrm{~A}$ recent trend has emerged among BPH patients towards medical treatment, such as alpha blockers (AABs) and 5alpha reductase inhibitors (5ARIs), over the former gold standard surgical procedure. ${ }^{1,17}$ In addition to the conventional medical management, the number of patients who choose phytotherapies (e.g., Serenoa repens, Pygeum africanum, pollen extracts) as complementary and alternative treatments is increasing steadily around the world. ${ }^{18-20}$
In Asian countries, especially China, Chinese herbal medicine (CHM) is commonly used as an alternative or complement to conventional medication and phytotherapy. ${ }^{21}$ Before the introduction of conventional medication and surgery to China, the Chinese had solely relied on CHM to treat BPH for more than three thousand years. However, the clinical efficacy and safety concerns of CHM in the treatment of BPH remain unclear. Although much of the classic literature, case series and trials have reported the clinical effects of different formulae of Chinese medicine, the therapeutic effects of $\mathrm{CHM}$ as a whole have not been evaluated. Therefore, we conducted a systematic review to evaluate the efficacy and adverse events of $\mathrm{CHM}$ in men with BPH.

This study aimed to evaluate the efficacy and side effects of Chinese herbal medicine versus placebo or active control in the treatment of symptomatic BPH.

\section{MATERIALS AND METHODS}

Criteria for considering studies for this review

Types of studies. Randomized, controlled clinical trials.

Types of participants. Men with LUTS consistent with BPH.

${ }^{1}$ The University of Hong Kong, School of Chinese Medicine, Hong Kong 852, China; ${ }^{2}$ The University of Hong Kong, Tung Wah Hospital, Hong Kong, China and ${ }^{3}$ Hospital Authority, Chinese Medicine Section, Hong Kong, China

Correspondence: Dr ZJ Zhang (zhangzj@hku.hk)

Received: 22 November 2012; Revised: 16 December 2012; Accepted: 5 January 2013; Published online: 3 June 2013 
Types of interventions. Comparison of oral CHM, either used alone or in adjuvant use with Western medication (WM), including phytomedicine, with WM and placebo for the treatment of $\mathrm{BPH}$.

Types of outcome measures. Primary outcomes. One of the primary outcomes was the International Prostate Symptom Score (IPSS), which ranges from 0 to 35 , with $0-7,8-19$ and higher than 20 designating mild, moderate and severe symptoms, respectively. Another primary outcome was the QoL score, which ranges from 0 to 6, with dissatisfaction increasing with the score. The effective rate was also considered as a primary outcome.

Secondary outcomes. The secondary outcomes included the maximum flow rate of urine (MFR, measured in $\mathrm{ml} \mathrm{s}^{-1}$ ), prostate volume $(\mathrm{PV}$, measured in $\mathrm{ml})$, residual urine volume ( $\mathrm{RU}$, measured in $\mathrm{ml}$ ) and adverse events (either drug related or all-cause).

\section{Search methods for the identification of studies}

We searched the MEDLINE, EMBASE, Cochrane Central Register of Controlled Trials, PubMed, ISI Web of Science, Scopus, Cumulative Index to Nursing and Allied Health Literature, Allied and Complementary Medicine, KoreaMed, J-STAGE and Google Scholar from inception to July 2011 using the grouped terms (BPH or nocturia or urination disorder) and (herb or herbal medicine or traditional Chinese medicine or TCM). The search also included China Journals Full-Text Database, China Masters and Doctoral Theses Full-text Database, China Proceedings of Conference Full-Text Database and Chinese Scientific Journal Database. The reference lists of all retrieved trials and previous reviews were searched for additional trials. Relevant peer-reviewed journals, conference proceedings, grey literature and ongoing and unpublished studies were also hand searched. There were no language restrictions.

\section{Data collection and analysis}

Two authors (CHM and WLL) searched the databases and identified the eligible citations independently. Disagreement about the eligibility of the included studies between the two authors was resolved by discussion. Consultation with a third reviewer (ZJZ) was sought when consensus was not achieved after the discussion. Excluded studies were also listed with exclusion reasons.

One author (CHM) extracted the data into Microsoft Excel spreadsheets by double entry. Discrepancies found in any one of the entries were amended with reference to the original text. Missing data were sought from the authors via e-mail. The extracted data were reviewed by another author (WLL). Any discrepancies were resolved by discussion.

The quality of the studies was assessed by Jadad scores. ${ }^{22}$ and Cochrane's risks of bias assessments. ${ }^{23}$ Points were awarded as follows: study described as randomized, 1 point; appropriate randomisation method, 1 point; study described as double-blinded, 1 point; appropriate double-blinded method, 1 point; and description of withdrawals and dropouts, 1 point. Points were also deducted as follows: sequence generation of randomisation was inappropriate, 1 point; and blinding method was inappropriate, 1 point. The Jadad scale score ranges from 1 to 5 , and studies scoring $\geqslant 3$ were considered to be of moderate quality. ${ }^{24}$

The risk of bias assessment examines the quality of the studies by seven domains: adequate sequence generation; allocation concealment; blinding of participants and personnel; blinding of outcome assessors; complete outcome data collection and reporting; lacking selective reporting; and free from other bias. Each domain was graded as high risk of bias, low risk of bias and unclear (uncertain risk). ${ }^{23}$
Our statistical analysis was performed by the Review Manager software (RevMan version 5.1. Copenhagen: The Nordic Cochrane Centre, The Cochrane Collaboration, 2011). ${ }^{25}$ For continuous outcomes, mean differences with $95 \%$ CIs were used. Standardized mean differences (SMDs) were used in cases combining unequal scales and units. For dichotomous outcomes, data were expressed as relative risk (RR) or absolute risk reduction (RD) with 95\% CI.

Meta-analysis for efficacy was performed on studies with a Jadad score $\geqslant 3$ and comparable clinical characteristics, while for adverse events, data from all included studies were analysed. The $I^{2}$ statistic was used to assess for heterogeneity among the included studies. A fixed effects model and a random effects model were used when statistical heterogeneity was not important $\left(I^{2}\right.$ statistics $\left.\leqslant 40 \%\right)$ or moderate to considerable $\left(I^{2}\right.$ statistics $\geqslant 40 \%$ ), respectively. ${ }^{25}$

We attempted to conduct subgroup analysis by the different types of WM used in the control group ( $\alpha$-blockers, 5-ARIs, phytomedicine) or as the adjuvant therapy in the experimental group.

When moderate or high levels of heterogeneity $\left(I^{2}>50 \%\right)$ were detected by the random-effects model, or if clinical or study design heterogeneity was judged to be present, a sensitivity analysis was conducted to assess the robustness of the pooled outcomes and conclusions of this review.

\section{RESULTS}

\section{Description of the paper selection process}

The search yielded 13922 potential citations for review, of which 11 915 were duplicates and 1785 were excluded for irrelevance. The full text of 222 articles was retrieved for detailed assessment, and 191 were further excluded for various reasons (Figure 1). The remaining 31 studies were included and analysed for the number of adverse events

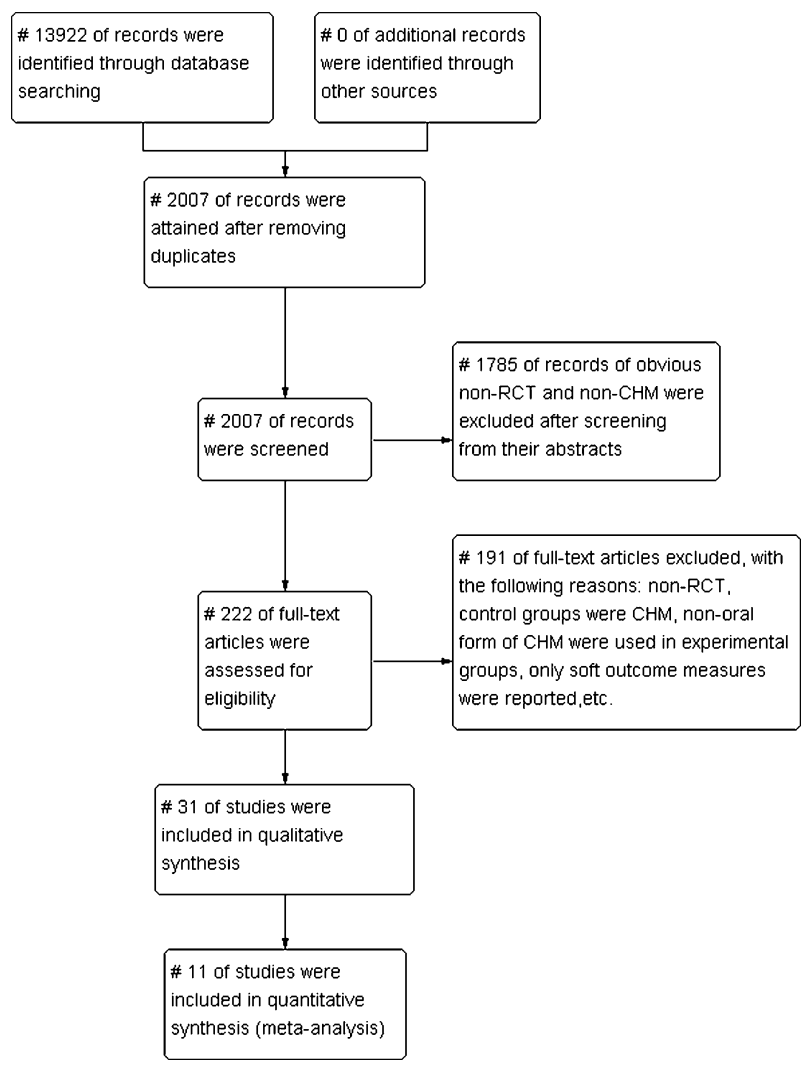

Figure 1 Selection of trials for inclusion in the review. 
and the use of herbs. Eleven with moderate to high study quality (Jadad score $\geqslant 3$ ) were included in this meta-analysis of the efficacy of CHM. Full details of the excluded studies are available from the authors upon request.

\section{Quality assessment by the Jadad scale}

Table 1 summarizes the study quality of the 31 included studies. Twenty studies $(64.5 \%)$ were rated as low-quality randomized controlled trials (RCTs) (Jadad score $\leqslant 2$ ), and the remaining $11(35.5 \%)$ were rated as moderate-to-high (Jadad score $\geqslant 3$ ). Table 2 presents the characteristics of the 11 moderate to high quality studies ${ }^{26-36}$ that were selected for detailed analysis. All 11 of the studies were reported as randomized trials; only one (Study 6$)^{33}$ did not describe the method of randomisation. Five trials fulfilled the criteria of a double-blind study design and provided an appropriate description of the double-blind method (Studies 3, 4, 5, 6 and 11). ${ }^{27,31-33,35}$ Only four studies described the dropouts and listed the reasons (Studies 4, 5, 6 and 9)..$^{27,28,32,33}$

\section{Assessment by the Cochrane's risks of bias assessment}

Figures 2 and 3 display the proportion and summary of the high, low and unclear risk of bias of 11 studies with Jadad scores $\geqslant 3$. Five studies were considered to have high risk of other bias (Studies 1, 7, 8, 9 and 11). In these studies, subjects were diagnosed with a certain type of TCM syndrome differentiation by the clinician. The assessment of subjects' pulse patterns and tongue outlooks relied on the clinicians' own judgment and hence might have involved subjective bias. Some domains in all studies were considered to have unclear risks of bias, as they were just mentioned in the article without detailed descriptions for bias assessment. All of the studies mentioned randomisation and the method of sequence generation, except for study 6 , which did not describe the sequence generation. Only studies 3, 4, 5 and 11 mentioned and described a proper method of allocation concealment. Five of the 11 studies (Studies 3, 4, 5, 6 and 11) mentioned blinding the participants and personnel, but none described the blinding of the evaluators. All studies mentioned either that all participants had completed the trial or provided the number of dropouts. Study 10 provided the outcome data as effective rates, but data for individual outcomes (IPSS and MFR) were not described; thus, there is a high risk of bias in selective reporting.

\section{Description of the studies}

The sample sizes of the 31 studies ranged from 40 to 160, comprising a total of 2493 subjects. The prostate volumes of the patients varied from 22.8 to $52.4 \mathrm{ml}$. Twenty-seven studies $(87.1 \%)$ were carried out in China and published in Chinese language journals from 1997 to 2011, while the remaining four studies were published in English- medium journals and conducted in Japan, Peru and India. Sixteen $(51.6 \%)$ of the 31 studies used WM or phytomedicine (PHY) as the basis for comparison, and two studies $(6.5 \%)$ used placebo control. The adjuvant use of CHM with WM or PHY was compared with WM or PHY alone in nine studies (29\%). Four studies (12.9\%) adopted a three- or four-arm study design (Table 1).

Of the 11 moderate-to-high quality studies selected for detailed analysis, CHM was compared with placebo alone in two studies (Studies 4 and 6), with tamsulosin alone in three studies (Studies 1, 5 and 8), with finasteride alone in one study (Study 10), and with both tamsulosin and finasteride in one study (Study 7). CHM was also compared with Saw Palmetto in one study (Study 11). Study 9 compared CHM plus tamsulosin with tamsulosin alone. Studies 2 and 5 had three (CHM, vitamin as placebo and Pygeum africanum) and four (three different doses of CHM and placebo) comparison groups, respectively.

The subjects' duration of BPH, as reported in the 11 studies, ranged from 15 days to 15 years. The criteria used for diagnosis of $\mathrm{BPH}$ were reported in four studies, including the clinical research guidelines of the new CHM, International Consultation Committee recommendations for the diagnosis and treatment of $\mathrm{BPH}$, Urological Surgery written by $\mathrm{Wu}$ Jie Ping and the guidelines for the diagnosis and treatment of BPH. Four studies further classified BPH into different TCM diagnostic patterns. ${ }^{37}$ Eight and two studies examined outpatients and mixed patients, respectively, and another one study did not mention whether the subjects were in- or outpatients (Table 2 ).

\section{Description of the CHM used}

Twenty-four standardized Chinese herbal formulae were examined in $23(74.2 \%)$ of the 31 studies, while the other eight studies used individualized approaches. Rou Gui (Cortex Cinnamomi) was the most frequently used single herb and was used in 17 (54.8\%) of the 31 studies. It was followed by Huang Qi (Radix Astragali) and Chuan Shan Jia (Manis pentadactyla L.) (41.9\%), Shu Di Huang (Radix Rehmanniae Perparata) (38.7\%), Shan Zhu Yu (Fructus Corni) and Fu Ling (Poria) (32.3\%), E Shu (Rhizoma Curcumae), Huang Bo (Cortex Phellodendri), Ze Xie (Rhizoma Alismatis) and Wang Bu Liu Xing (Semen Vaccariae) (29.0\%) (Table 3). Fifteen $(46.9 \%)$ of the 32 herbal formulae used in the studies were prepared in decoction; $12(37.5 \%)$ were manufactured in capsules; $3(9.4 \%)$ in tablets; 1 $(3.1 \%)$ in pill; and the remaining one $(3.1 \%)$ in a combination of decoction and pills. The selected CHM was taken one to three times per day, and the treatment courses ranged from 1 to 12 months.

Efficacy assessment. The 11 studies with Jadad scores $\geqslant 3$ were selected for detailed assessment and meta-analysis. Five of the 11 moderate-tohigh quality studies (Studies 1, 5, 6, 10 and 11) reported the effective

Table 1 Study design and quality of the included trials

\begin{tabular}{|c|c|c|c|c|c|c|c|c|}
\hline & \multirow{2}{*}{ No. of studies } & \multirow{2}{*}{ No. of randomized subjects } & \multicolumn{5}{|c|}{ Total Jadad score } & \multirow{2}{*}{$\begin{array}{c}\text { No. of studies with } \\
\text { Jadad score } \geqslant 3\end{array}$} \\
\hline & & & 1 & II & III & IV & $V$ & \\
\hline CHM vs. placebo & 2 & 224 & 0 & 0 & 0 & 1 & 1 & 2 \\
\hline CHM vs. WM & 16 & 1225 & 1 & 9 & 4 & 0 & 2 & 6 \\
\hline $\mathrm{CHM}+\mathrm{WM}$ vs. WM & 9 & 819 & 1 & 7 & 1 & 0 & 0 & 1 \\
\hline Three- or four-arm design & 4 & 290 & 1 & 1 & 1 & 0 & 1 & 2 \\
\hline Total & 31 & 2558 & 3 & 17 & 6 & 1 & 4 & 11 \\
\hline$\%$ & 100 & 100 & 9.7 & 54.8 & 19.4 & 3.2 & 12.9 & 35.5 \\
\hline
\end{tabular}

Abbreviations: CHM, Chinese herbal medicine; WM, Western medication. 


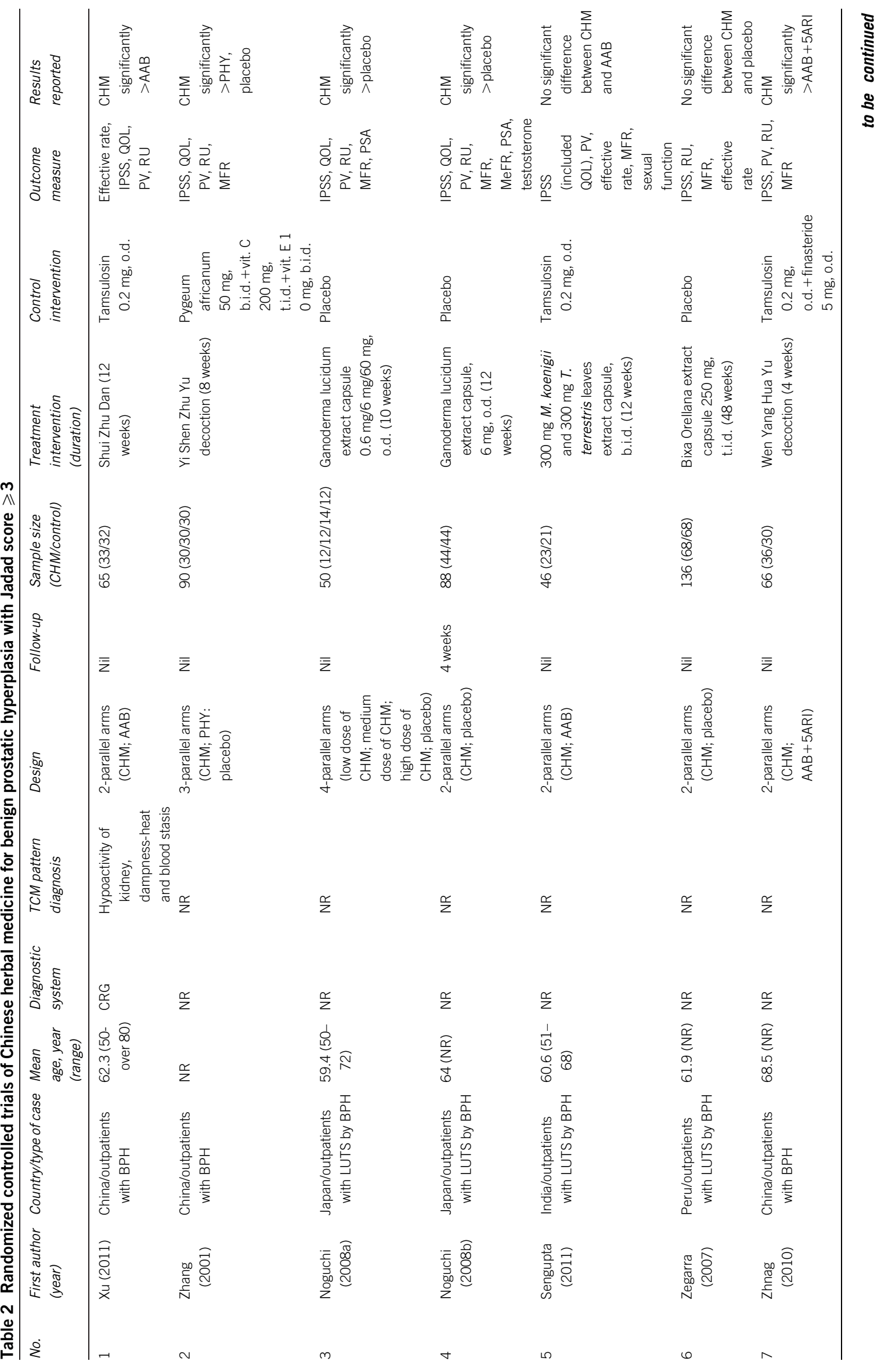




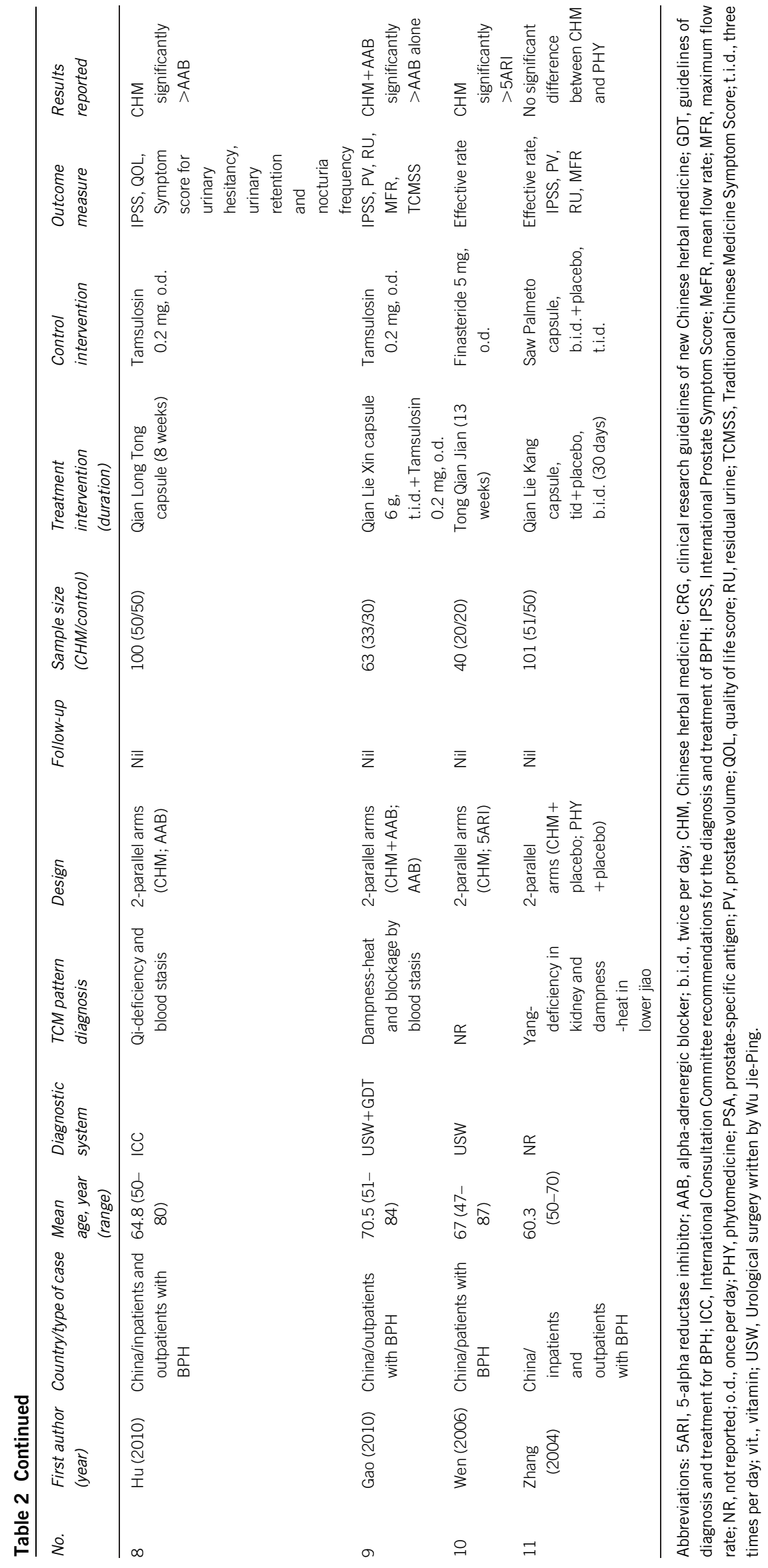




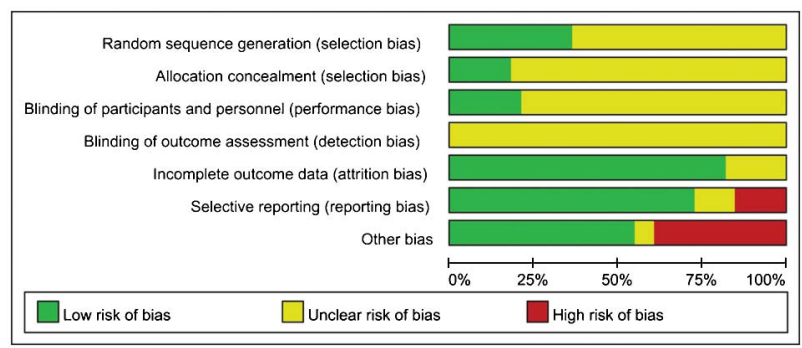

Figure 2 Risk of bias graph.

rate, and it was the only outcome measure reported in study 10 . The definition of effective rate was not standardized. Highly effective rate was most often defined as the proportion of subjects who had any two of the following items: IPSS $\leqslant 7$ points, QoL $\leqslant 1$ point, $90 \%$ or above reduction in total score of disease progression, below $60 \%$ of the original PV or MFR $\geqslant 18 \mathrm{ml} \mathrm{s}^{-1}$. Effective rate was defined as the proportion of patients who experienced any one of the following items: $7 \leqslant$ IPSS $\leqslant 13$ points, QoL dropped from $4-6$ points to $2-$ 3 points, $60 \%-90 \%$ reduction in total score of disease progression, $60 \%-80 \%$ of the original PV, MFR $\geqslant 12 \mathrm{ml} \mathrm{s}^{-1}$ or more than $50 \%$ reduction in $\mathrm{RU}^{37}$ The effective rates of $\mathrm{CHM}$ derived from the five studies varied from $43.5 \%$ to $100 \%$; for the placebo group, from $30.9 \%$ to $55.9 \%$; and for the AAB or $5 \mathrm{ARI}$ group, from $33.3 \%$ to $95 \%$. IPSS and QoL were reported as the subjective outcome measures in all of the 11 studies except in one (Study 10) and five (Studies 1, 2, 3, 4 and 8) studies, respectively. PV, MFR and RU were used as the objective outcome measures in nine (Studies 1, 2, 3, 4, 5, 6, 7, 9 and 11), eight (Studies 2, 3, 4, 5, 6, 7, 9 and 11) and eight studies (Studies 1, 2, 3, 4, 6, 7 and 11), respectively. All 11 studies assessed only the short-term effect of CHM (Table 2).

CHM versus placebo. CHM was reported to be more effective than placebo in three studies (Studies 2, 3 and 4), but Study 6 did not show any difference between the two groups $(P>0.05)$. The meta-analysis of these four studies did not detect any significant between-group difference in improving MFR and PV $(P>0.05)$. Though the pooled analysis of IPSS, QoL and RU also did not show any group difference $(P<0.05)$, subgroup analyses of post-treatment measurements were found favouring CHM in IPSS (SMD: $-2.93,95 \% \mathrm{CI}:-3.82$ to -2.04 , $P<0.00001$ ), QoL (SMD: $-0.75,95 \%$ CI: -1.22 to $-0.29, P=0.002$ ) and RU (SMD: $-0.83,95 \% \mathrm{CI}:-1.47$ to $-0.18, P=0.01$ ). The only study with an effective rate measure (Study 6) showed that there was no difference between $\mathrm{CHM}$ and placebo in improving the symptoms score (RR: 0.97, RD: -0.015 (95\% CI: $-0.18-0.15)$ ), PV (RR: 0.85, RD: -0.059 (95\% CI: $-0.21-0.10)$ ) or RU (RR: 1.14, RD: $0.044(95 \%$ CI: $-0.11-0.20)$ ).

CHM versus WM. CHM was compared with Western medication in seven studies, with three studies using tamsulosin (Studies 1, 5 and 8), one study using finasteride (Study 10), one study using tamsulosin and finasteride (Study 7), one study using Pygeum africanum (Study 2) and one study using Saw Palmetto (Study 11). The pooled analysis did not show any difference in the effective rates between the CHM and WM groups (RR: $1.06,95 \% \mathrm{CI}: 0.92$ to $1.22, I^{2}=33 \%, P=0.42$ ), while $\mathrm{CHM}$ was found to be superior to AABs only in sub-group analysis (RR: 1.27, 95\% CI: $\left.1.01-1.60, I^{2}=0 \%, P=0.04\right)$. In addition to the effective rate, insignificant differences were found in IPSS, MFR and RU improvement $(P>0.05)$. However, pooled analysis showed that CHM was

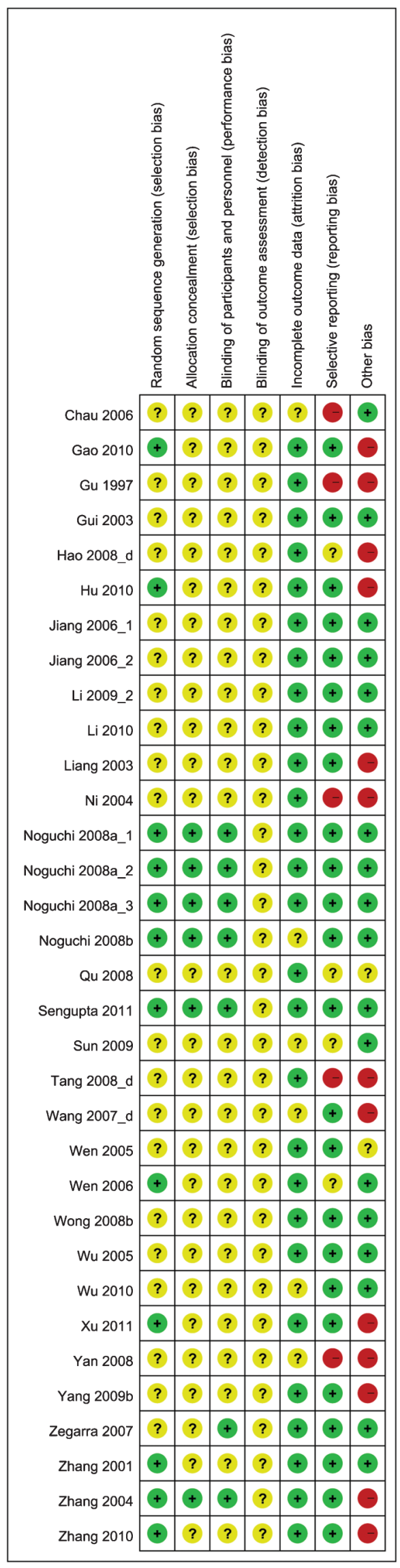

Figure 3 Risk of bias summary. 
Table 3 The 10 most frequently used herbs for BPH in the 31 reviewed studies

\begin{tabular}{llr}
\hline Chinese name & English name & N (\%) \\
\hline Rou Gui & Cortex Cinnamomi & $17(54.8)$ \\
Huang Qi & Radix Astragali & $13(41.9)$ \\
Chuan Shan Jia & Manis pentadactyla & $13(41.9)$ \\
& $\quad$ Linnaeus & \\
Shu Di Huang & Radix Rehmanniae & $12(38.7)$ \\
& $\quad$ Perparata & \\
Shan Zhu Yu & Fructus Corni & $10(32.3)$ \\
Fu Ling & Poria & $10(32.3)$ \\
E Shu & Rhizoma Curcumae & $9(29.0)$ \\
Huang Bo & Cortx Phellodendri & $9(29.0)$ \\
Ze Xie & Rhizoma Alismatis & $9(29.0)$ \\
Wang Bu Liu Xing & Semen Vaccrriae & $9(29.0)$ \\
\hline
\end{tabular}

superior to WM in improving QoL scores (SMD: $-1.07, I^{2}=40 \%$, $P<0.00001$ ) (Figure 4) and reducing PV (SMD: $-0.31, I^{2}=30 \%$, $P=0.03$ ) (Figure 5).

CHM plus WM versus WM alone. Only one study (Study 9) compared the adjuvant use of $\mathrm{CHM}$ and WM with WM alone. It was found that CHM plus tamsulosin $\left(0.2 \mathrm{mg} \mathrm{day}^{-1}\right)$ was superior to tamsulosin $0.2 \mathrm{mg} \mathrm{day}^{-1}$ alone in reducing the post-treatment TCM symptom score (mean difference: $-7.9,95 \%$ CI: -9.75 to $-6.05, P<0.0001$ ). There were no differences in IPSS, PV, MFR or RU improvement between the two groups $(P>0.05)$. After performing sensitivity analyses for all comparisons, the results for all outcomes remained robust.

Adverse event reporting. Adverse events were reported in 17 (54.8\%) of the 31 reviewed studies, but only 15 studies provided the

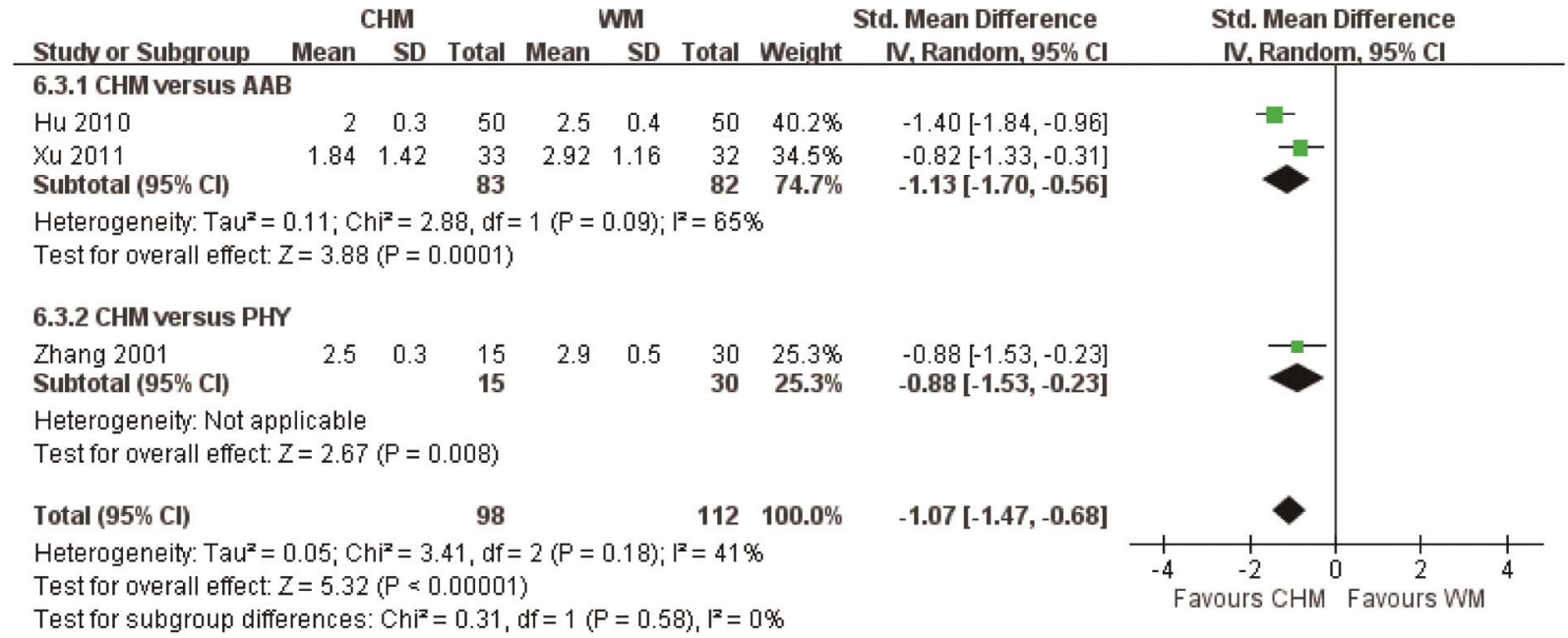

Figure 4 Quality of life when using Chinese herbal medicine (CHM) and Western medicine (WM) for benign prostatic hyperplasia. AAB, alpha-adrenergic blocker; PHY, phytomedicine.

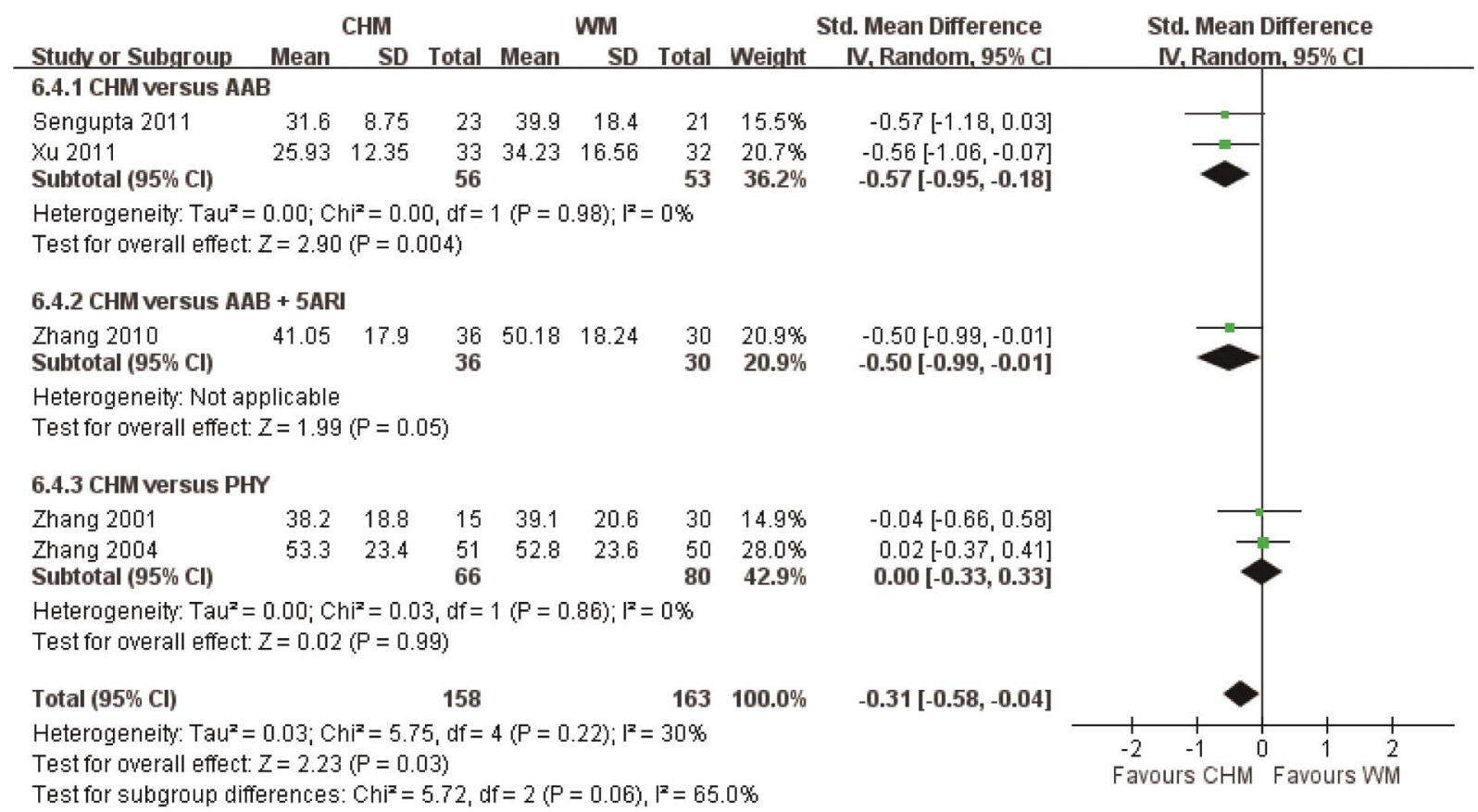

Figure 5 Prostate volume when using Chinese herbal medicine (CHM) and Western medicine (WM) for benign prostatic hyperplasia. AAB, alpha-adrenergic blocker; PHY, phytomedicine; 5ARI, 5-alpha reductase inhibitor. 


\begin{tabular}{|c|c|c|c|c|c|c|c|c|c|}
\hline Study or Subgroup & $\begin{array}{l}\text { CHM } \\
\text { Events }\end{array}$ & Total & $\begin{array}{l}\text { Placel } \\
\text { Events }\end{array}$ & Total & Weight & $\begin{array}{c}\text { Risk Ratio } \\
\text { M-H. Fixed. } 95 \% \mathrm{Cl}\end{array}$ & & $\begin{array}{r}\text { Risk } \\
\text { M-H. Fixe }\end{array}$ & $\begin{array}{l}\text { Ratio } \\
\text { ed. } 95 \% \mathrm{Cl}\end{array}$ \\
\hline Noguchi 2008a_1 & 3 & 12 & 1 & 4 & $8.9 \%$ & $1.00[0.14,7.10]$ & & & \\
\hline Noguchi 2008a_2 & 1 & 12 & 1 & 4 & $8.9 \%$ & $0.33[0.03,4.19]$ & & & \\
\hline Noguchi 2008a_3 & 2 & 14 & 1 & 4 & $9.2 \%$ & $0.57[0.07,4.80]$ & & & \\
\hline Noguchi 2008b & 6 & 44 & 11 & 44 & $65.1 \%$ & $0.55[0.22,1.35]$ & & & \\
\hline Zegarra 2007 & 2 & 68 & 1 & 68 & $5.9 \%$ & $2.00[0.19,21.54]$ & & & \\
\hline Zhang 2001 & 1 & 15 & 0 & 30 & $2.0 \%$ & $5.81[0.25,134.73]$ & & & \\
\hline Total $(95 \% \mathrm{Cl})$ & & 165 & & 154 & $100.0 \%$ & $0.76[0.40,1.46]$ & & & \\
\hline Total events & 15 & & 15 & & & & & & \\
\hline $\begin{array}{l}\text { Heterogeneity: } \mathrm{Chi}^{2}= \\
\text { Test for overall effect }\end{array}$ & $\begin{array}{l}32, \mathrm{df}=5 \\
=0.82(P\end{array}$ & $\begin{array}{l}(P=0 \\
=0.4\end{array}$ & 1) 65$) ; 1^{2}=$ & & & & $\begin{array}{l}0.005 \\
\text { Fav }\end{array}$ & $\begin{array}{l}0.1 \\
\text { ours } \mathrm{CHM}\end{array}$ & $\begin{array}{l}10 \\
\text { Favours }\end{array}$ \\
\hline
\end{tabular}

Figure 6 Likelihood of adverse events when using Chinese herbal medicine (CHM) and placebo.

frequency of these adverse events. Meta-analysis of the four studies that compared CHM with placebo showed that there were no group differences in the occurrence of adverse events (RR: 0.50, 95\% CI: $0.32-0.78, I^{2}=56 \%, P=0.41$ ) (Figure 6). A pooled analysis of eight studies comparing CHM with WM showed that WM has a higher frequency of adverse events (RR: 0.76, 95\% CI: 0.40-1.46,
$I^{2}=0 \%, P=0.003$ ) (Figure 7). Four of the eight studies used alphaadrenergic blockers, one study used 5ARIs, two studies used phytomedicine and one study used a combination of an alpha-adrenergic blocker and a 5ARI for comparison. Meta-analysis of the four studies comparing the adjuvant use of CHM and WM with WM alone showed that there were no group differences in the

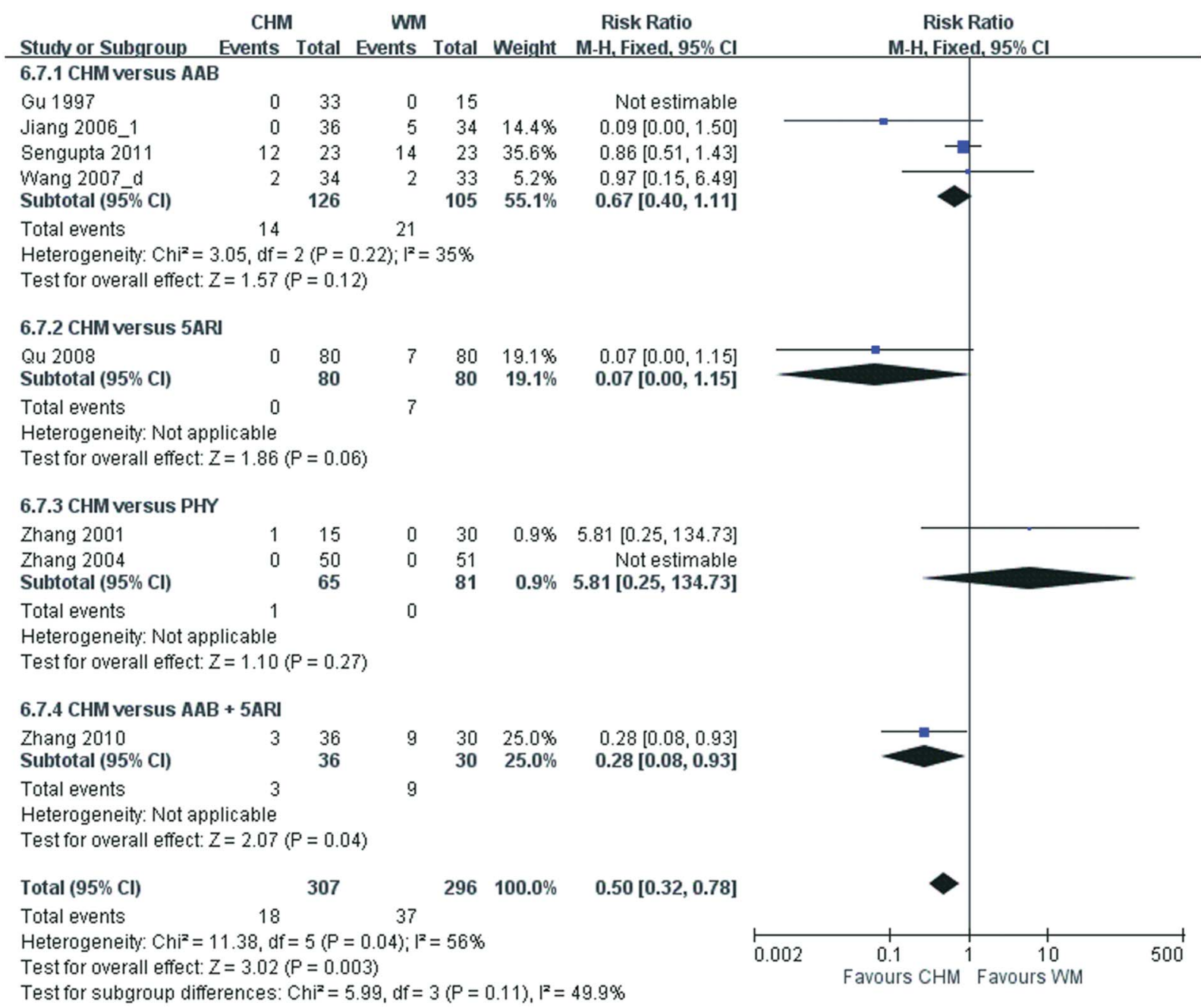

Figure 7 Likelihood of adverse events when using Chinese herbal medicine (CHM) and Western medicine (WM). AAB, alpha-adrenergic blocker; PHY, phytomedicine; 5ARI, 5-alpha reductase inhibitor. 
occurrence of adverse events (RR: $1.56,95 \%$ CI: $0.53-4.60, I^{2}=0 \%$, $P=0.42$ ) (Figure 8).

Table 4 summarizes all of the reported adverse events. Regarding individual events, only nausea (RR: $0.07,95 \% \mathrm{CI}: 0.01-0.55$, two studies, $I^{2}=0 \%$ ) and dizziness (RR: $0.25,95 \%$ CI: $0.06-0.95$, three studies, $I^{2}=37 \%$ ) were found to be more frequent in patients taking WM than those on CHM. Meta-analysis of the frequencies of the adverse events from the eight studies that compared CHM with placebo were found to be similar to those comparing the adjuvant use of $\mathrm{CHM}$ and WM with WM alone.

\section{DISCUSSION}

This study is the first systematic review of English and Chinese literature to investigate the efficacy and safety of CHM for BPH. The frequencies of the commonly used herbs for BPH were examined. The overall result from the 31 included RCTs suggested that CHM and its adjuvant use with WM were superior to $\mathrm{WM}$ alone and to the placebo in the treatment of $\mathrm{BPH}$, but the meta-analyses of the moderate-tohigh quality studies could not provide evidence for this conclusion.

Although the PICOS criteria (patients, intervention, comparison, outcomes and study design) were adequately addressed in the included studies in general, their means of sequence generation for randomisation, allocation concealment and blinding of subjects, investigators and collectors were rarely reported. Thus, most of the RCTs reviewed were scored 2 or less by the Jadad scale and deemed to be of low quality. Even for the 11 RCTs that scored 3 or above, five of them had at least one domain with a high risk of bias, according to the Cochrane assessment criteria.

The overall completeness of the evidence was only fair. For the meta-analysis of the 11 moderate-to-high quality studies, there were relatively sufficient trials comparing CHM to WM $(n=7)$. However, there were insufficient trials for comparing CHM to placebo $(n=4)$ and $\mathrm{CHM}+\mathrm{WM}$ to WM $(n=1)$. Nearly all of the included studies did not report the herbs' quality or the preparation procedure of the herbal formulas; therefore, the ability to replicate the CHM and treatment integrity were not assured. Calculations of sample size and statistical power were not reported in the studies. The sample sizes of some studies were less than 30 in each group, which may not be strong enough to detect significant group differences. Other methodological limitations included subjective bias in TCM diagnosis by practitioners and insufficient adverse event reporting. In Western countries, tamsulosin is normally used at $0.4 \mathrm{mg}$ doses for $\mathrm{BPH}$. However, only one study ${ }^{27}$ used $0.4 \mathrm{mg}$ tamsulosin for comparison. The other five studies $^{26,28-30,38}$ used a half dose $(0.2 \mathrm{mg})$ of tamsulosin instead; thus, the result might not truly reflect the efficacy and adverse events of tamsulosin in the usual clinical setting. Although the self-reported QoL scores were measured by the same validated questionnaire, the timing of the assessment differed within the included studies. Because nearly one-third of the included studies did not provide the age range of the patients, the sample could not be defined as homogeneous by age. The diagnosis criteria of BPH were not standardized, and the follow-up periods were either not specified or were specified, but were not long enough among the included studies. These shortcomings might obscure the fair comparison of these studies.

The use of CHM as monotherapy and adjuvant therapy was diversified in the formula composition and dosage of the included studies. Such a large difference in herbal combinations may be due to the complexity of TCM diagnosis and the personal experiences of practitioners. Based on the symptoms and signs of individual patients, TCM practitioners would classify them into different syndrome differentiations (Zheng in Chinese) and prescribe herbal formulas accordingly. ${ }^{39}$ Some complementary herbs would be added if necessary. Therefore, even trials with exactly the same $\mathrm{CHM}$ formulas and dosages may not simulate the usual practice of TCM, thus rendering proving the efficacy of a particular formula impossible.

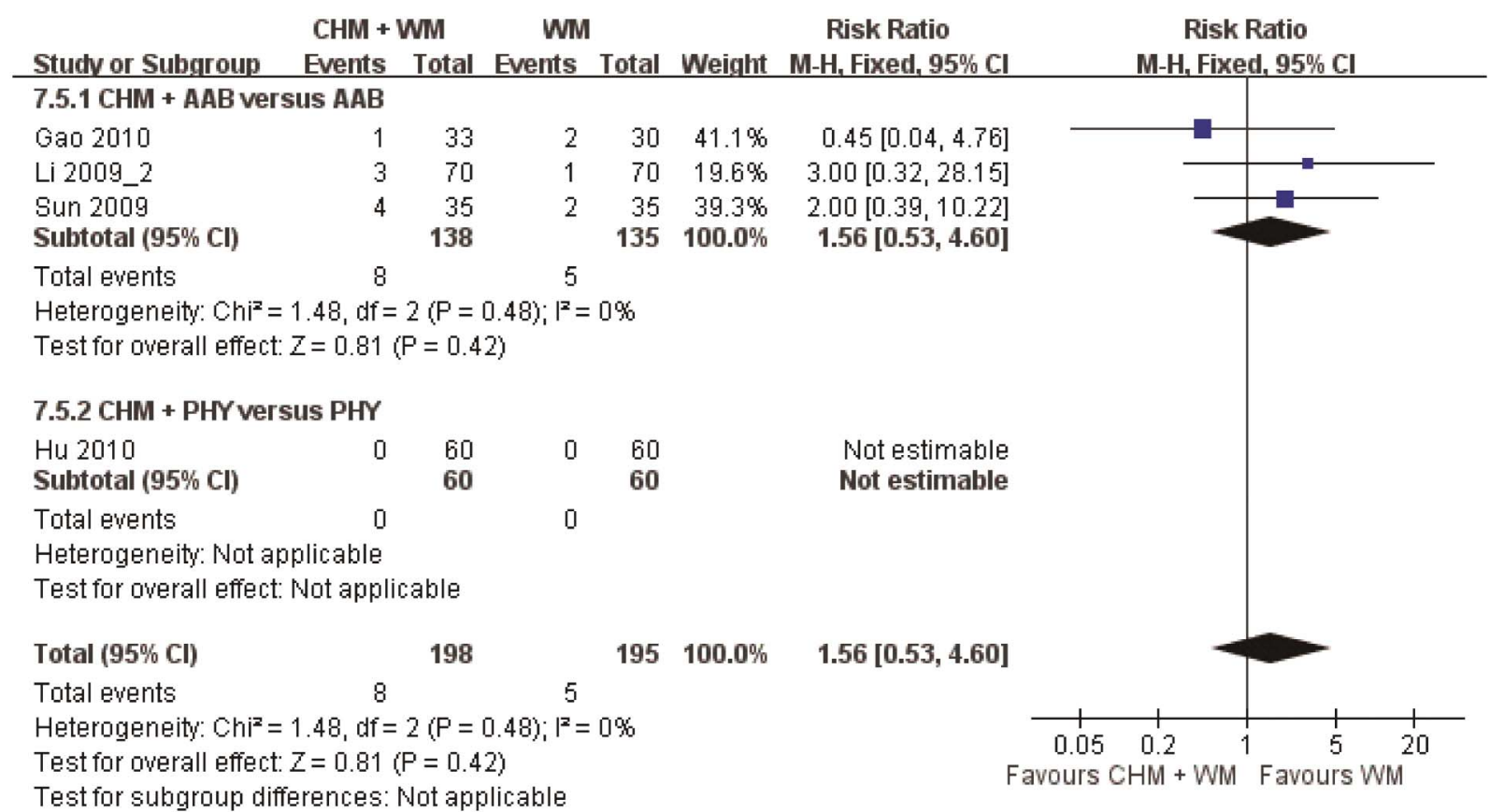

Figure 8 Likelihood of adverse events with the adjuvant use of Chinese herbal medicine (CHM) and Western medicine versus (WM) Western medicine alone. AAB, alpha-adrenergic blocker; PHY, phytomedicine. 
Table 4 Risk ratio of all reported adverse events in studies comparing CHM with placebo, with WM and in studies comparing CHM+WM with WM alone

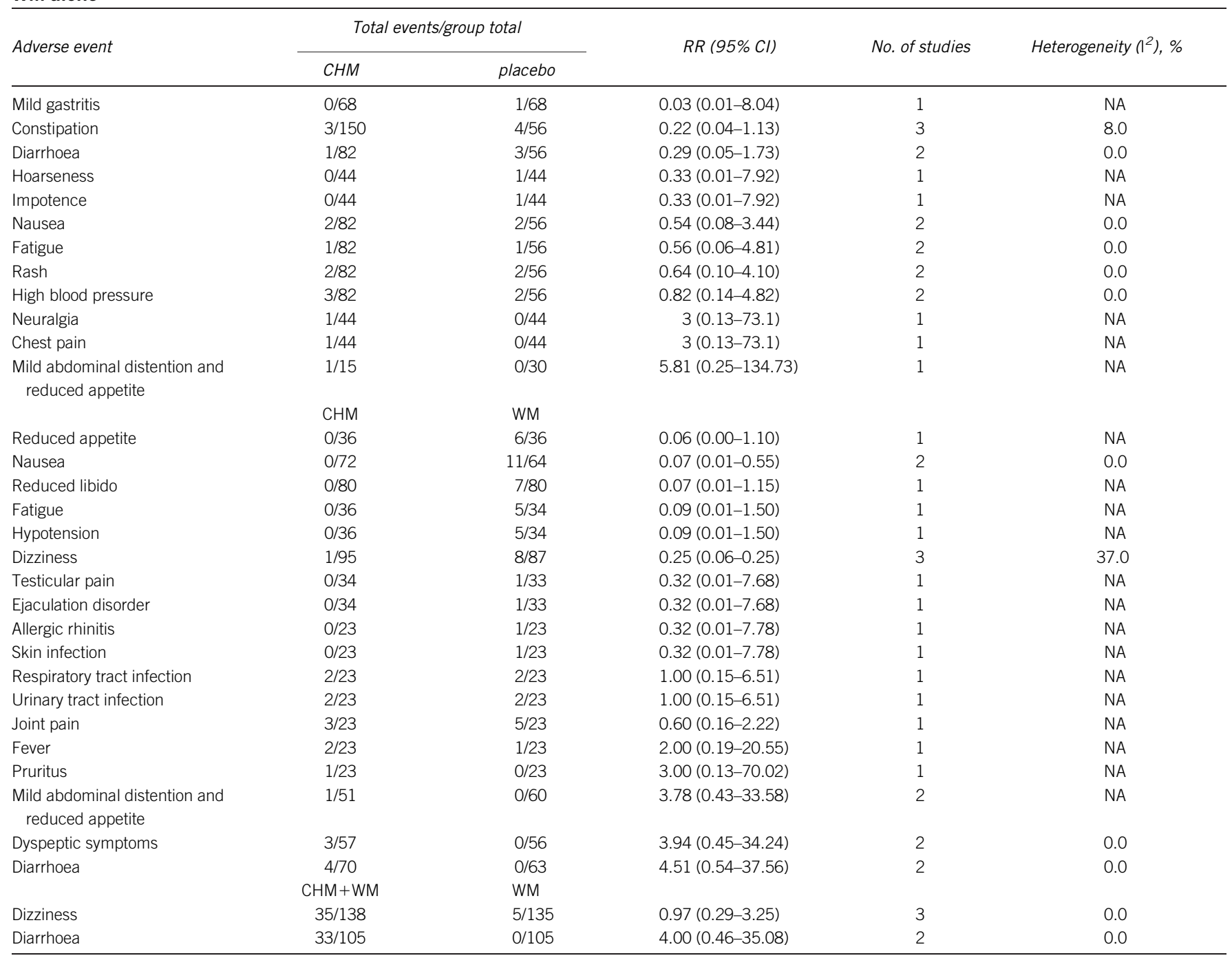

Abbreviations: CHM, Chinese herbal medicine; NA, not applicable; RR, risk ratio; WM, Western medication.

From the point of view of traditional Chinese medicine (TCM), $\mathrm{BPH}$ LUTS is equivalent to the 'Long Bi' (obstructed urination), ${ }^{40}$ and its hyperplasic nature can be classified into the category of 'Zheng Jia' (tumour) of TCM. ${ }^{41}$ TCM believes that the Yang deficiency in the kidney and blood stasis are the main syndrome differentiations of 'Long Bi' and 'Zheng Jia'. Thus, tonifying the Yang in the kidney and removing the blood stasis are considered to be the major strategy to treat 'Long Bi' and 'Zheng Jia'.

Among the 31 included studies, Cortex Cinnamomi $(n=17)$ was the most frequently used herb, likely because it is considered to be able to tonify the Yang in the kidney by TCM practitioners. Radix Astragali $(n=13)$ was found to be the second most commonly used herb, which is believed to be a strong Qi tonifying herb. Manis pentadactyla L. ( $n=13$ ) was the second, while both Rhizoma Curcumae $(n=9)$ and Semen Vaccariae $(n=9)$ were the fifth most frequently used herbs. These herbs are considered to be blood circulation enhancers and blood stasis removers. Because TCM believes that Qi is the driving force of blood circulation, Qi deficiency will lead to blood stasis. Therefore, these herbs can be used to restore the driving force for blood circulation and hence remove the stasis. Radix Rehmanniae
Perparata $(n=13)$ and Fructus Corni $(n=10)$ were the third and fourth most commonly used herbs, respectively, as they are considered to be agents to tonify the Ying in the kidney. According to TCM theory, developing a Yang deficiency in the kidney is a long-term process that is usually initiated by a Ying deficiency in the kidney. Ying is also another vital element for controlling kidney function, and its deficiency would cause other types of symptoms. Because the Ying and Yang in the kidney are mutually dependent, both should be tonified for a better outcome.

Poria Cortex $(n=10)$, Cortex Phellodendri $(n=9)$ and Rhizoma Alismatis $(n=9)$ were the fourth through sixth most commonly used herbs. These are considered to have the ability to remove dampness and to clear heat in the lower part of body, which is considered another important syndrome differentiation in 'Long $\mathrm{Bi}^{40}$ and is associated with urinary tract infection in BPH patients.

On top of the TCM theory, some pharmacological studies provided scientific evidence to elaborate on the possible mechanisms of the most commonly used herbs on $\mathrm{BPH}$. These herbs were found to be able to diminish the prostate size in rats by increasing the expression of inducible nitric oxide synthase in the prostate gland, hence inducing 
cell apoptosis. ${ }^{42}$ Radix Astragali was found to possess a moderate diuretic effect. ${ }^{43}$ Radix Astragali, ${ }^{44}$ Manis pentadactyla L. ${ }^{45}$ and Rhizoma Curcumae ${ }^{46}$ were found to be able to improve the haematological rheology. Semen Vaccariae was found to have anti-tumour activity, ${ }^{47}$ and Cortex Phellodendri was found to possess anti-bacterial and anti-viral effects. ${ }^{48}$

Meta-analyses of the 11 moderate-to-high quality studies with Jadad scores of 3 or above found that CHM was similar to placebo and WM, while only CHM was found to be superior to WM in improving QoL and reducing $\mathrm{PV}$ among $\mathrm{BPH}$ patients. The adjuvant use of $\mathrm{CHM}$ was also similar to WM alone in treating $\mathrm{BPH}$. Due to the small number of trials included and the heterogeneity existing across the studies, the results of this meta-analysis should be treated cautiously. Moreover, the duration of the included studies just ranged from 1 month to 1 year. In daily practice, BPH patients usually take either CHMWM or both for much longer time periods, even throughout their lives. Thus, the actual long-term effects and adverse events may not be reflected in the pooled analysis.

The frequency of adverse events was similar between CHM and placebo but higher with WM. The severities of the adverse events were either not reported or were reported as mild, and chest pain was the single serious adverse event reported. The combination of CHM and WM was similar to WM in the frequency of adverse events. CHM may be safe for application as a monotherapy or an adjuvant therapy in research and clinical settings. The diversity of CHM used in the included studies made our results difficult to be directly applied in clinical practice. To cope with this challenge, we presented the most frequently used herbs that we concluded may provide the various beneficial effects. Chinese Medicine Practitioners and researchers should consider and apply them in their clinical and research settings, respectively.

In conclusion, this review showed that CHM, either as monotherapy or an adjuvant therapy with WM, was similar to either placebo or WM in the treatment of BPH. Only CHM was found to be superior to $\mathrm{WM}$ in improving QoL and reducing PV. The frequency of adverse events of CHM was similar to that of placebo and even less than that of WM. Adjuvant use of CHM and WM was also similar to WM in the frequency of adverse events reported. Thus, CHM may be safe for use in research and clinical practice. Because the quality of the majority of the included studies was poor and the data were non-homogenous, the current evidence was not strong enough to support the definite advantage of CHM over other control groups. Therefore, this review should be treated as a pilot review to provide more reliable data for further studies. More trials of good quality and larger subject numbers should be conducted to further examine the result of this review. Finally, as CHM was analysed as a whole in this review, these results cannot be simply applied to every instance of single herbs or formulas.

\section{AUTHOR CONTRIBUTIONS}

CHM participated in the design of the study, collected the data, performed statistical analyses and drafted the manuscript. WLL collected and reviewed the data. ZJZ, SLL, XYC, VTW and EZ developed the conception of the study, gave their professional comments to the data and manuscript, and gave final approval of the version to be published. All authors read and approved the final manuscript.

\section{COMPETING FINANCIAL INTERESTS}

All authors declare that there are no competing financial interests.

\section{ACKNOWLEDGMENTS}

The present study was supported by the Hospital Authority of Hong Kong, China (HA 820/126/19/50/1).

1 Wei JT, Calhoun E, Jacobsen SJ. Benign prostatic hyperplasia. In: Litwin MS, Saigal CS, editors. Urologic Diseases in America. Washington, DC: NIH Publication; 2007. p43-67.

2 Barry MJ, Williford WO, Chang Y, Machi M, Jones KM et al. The American Urological Association Symptom Index for benign prostatic hyperplasia. J Urol 1992; 148: 1549-57.

3 Jacobsen SJ, Guess HA, Panser LA, Girman CJ, Chute CG et al. A population-based study of healthcare-seeking behavior for treatment of urinary symptoms. The Olmsted County Study of Urinary Symptoms and Health Status among Men. Arch Fam Med 1993; 2: 729-35.

4 Jacobsen SJ, Girman CJ, Guess HA, Oesterling JE, Lieber MM. New diagnostic and treatment guidelines for benign prostatic hyperplasia. Potential impact in the United States. Arch Intern Med 1995; 155: 477-81.

5 Arrighi HM Metter EJ, Guess HA, Fozzard JL. Natural history of benign prostatic hyperplasia and risk of prostatectomy: The Baltimore Longitudinal Study of Aging. Urology 1991; 38(Suppl 1): 4-8.

6 Norman RW, Nickel JC, Fish D, Pickett SN. 'Prostate-related symptoms' in Canadian men 50 years of age or older: prevalence and relationships among symptoms. Br J Urol 1994; 74: 542-50.

7 Garraway WM, Collins GN, Lee RJ. High prevalence of benign prostatic hypertrophy in the community. Lancet 1991; 338: 469-71.

8 Berges RR, Pientka L. Management of the BPH syndrome in Germany: who is treated and how? Eur Urol 1999; 36: 21-7.

9 Sagnier PP, MacFarlane G, Teillac P, Botto H, Richard F et al. Impact of symptoms of prostatism on level of bother and quality of life of men in the French community. J Urol 1995; 153: 669-73.

10 Bosch J, Hop WC, Kirkels WJ, Schröder FH. The International Prostate Symptom Score in a community-based sample of men between 55 and 74 years of age: prevalence and correlation of symptoms with age, prostate volume, flow rate and residual urine volume. Br J Urol 1995; 75: 622-30.

11 Wolfs G, Knottnerus JA, Janknegt RA. Prevalence and detection of micturition problems among 2,734 elderly men. J Urol 1994; 152: 1467-70.

12 Homma Y, Kawabe K, Tsukamoto T, Yamanaka H, Okada K et al. Epidemiologic survey of lower urinary tract symptoms in Asia and Australia using the international prostate symptom score. Int J Urol 1997; 4: 40-6.

13 Tsukamoto T, Kumamoto Y, Masumori N, Miyakr H, Rhodes T et al. Prevalence of prostatism in Japanese men in a community-based study with comparison to a similar American study. J Urol 1995; 154: 391-5.

14 Lee E, Yoo KY, Kim Y, Shin Y, Lee C. Prevalence of lower urinary tract symptoms in Korean men in a community-based study. Eur Urol 1998; 33: 17-21.

15 Gu FL. Epidemiological survey of benign prostatic hyperplasia and prostatic cancer in China. Chin Med J 2000; 113: 299-302.

16 Wong SY, Woo J, Hong A, Leung JC, Kwok T et al. Risk factors for lower urinary tract symptoms in southern Chinese men. Urology 2006; 68: 1009-14.

17 Litwin MS, Saigal CS. Introduction. In: Litwin MS, Saigal CS, editors. Urologic Diseases in America. Washington, DC: NIH Publication. p3-7.

18 Bales GT, Christiano AP, Kirsh EJ, Gerber GS. Phytotherapeutic agents in the treatment of lower urinary tract symptoms: a demographic analysis of awareness and use at the University of Chicago. Urology 1999; 54: 86-9.

19 Di Silverio F, Flammia GP, Sciarra A, Caponera M, Mauro M et al. Plant extracts in BPH. Minerva Urol Nefrol. 1993; 45: 143-9.

20 Buck AC. Phytotherapy for the prostate. Br J Urol 1996; 78: 325-36.

21 Zhou Z, Zhang XH, Yu PL, Dong BR, Jian ZJ. Current status of diagnosis and treatment for benign prostatic hyperplasia in geriatrics outpatient. Chin J Geriatr 2011; 30: 965-7.

22 Jadad AR, Moore RA, Carroll D, Jenkinson C, Reynolds DJ et al. Assessing the quality of reports of randomized clinical trials: is blinding necessary? Controlled Clin Trials 1996; 17: 1-12.

23 Higgins JPT, Altman DG, Sterne JAC (editors). Chapter 8: Assessing risk of bias in included studies. In: Higgins JPT, Green S (editors). Cochrane Handbook for Systematic Reviews of Interventions Version 5.1.0 (Updated March 2011). The Cochrane Collaboration, 2011. Available from HYPERLINK "http://www.cochranehandbook.org".

24 Manchikanti L, Ailinani H, Koyyalqunta D, Datta S, Singh V et al. A systematic review of randomized trials of long-term opioid management for chronic non-cancer pain Pain Physician 2011; 14: 91-121.

25 Deeks JJ, Higgins JPT, Altman DG (editors). Chapter 9: Analysing data and undertaking meta-analyses. In: Higgins JPT, Green S (editors). Cochrane Handbook for Systematic Reviews of Interventions Version 5.1.0 (updated March 2011). The Cochrane Collaboration, 2011. Available from HYPERLINK "http://www.cochranehandbook.org".

26 Xu ZM, Chen XY, Yang CJ, Yang ZW. Chinese medicine "Shui zhu dan" for 33 cases of benign prostatic hyperplasia treatment. J Tradit Chin Med 2011; 52 $1054-5$. 
27 Sengupta G, Hazra A, Kundu A, Ghosh A. Comparison of Murraya koenigii- and Tribulus terrestris-based oral formulation versus tamsulosin in the treatment of benign prostatic hyperplasia in men aged $>50$ years: a double-blind, doubledummy, randomized controlled trial. Clin Thers 2011; 33: 1943-52.

28 Gao Z, Lun LJ, Shao KQ, Shen JW, Li Q et al. Clinical observation on Qian Lie Xin capsule plus tamsulosin hydrochloride sustained release capsules in the treatment of benign prostatic hyperplasia with Shi Re Yu Zu syndrome. J TCM Univ Hunan 2011; 31: 17-20.

29 Zhang ZZ. Treatment of 36 cases of benign prostatic hyperplasia by Wan Yang Hua Yu decoction. Chin J Exp Tradit Med Form 2010; 16: 256-7.

$30 \mathrm{Hu} \mathrm{JH}$, Yuan YF, Yuan B, He JQ. Effect of Qian Long Tong capsule on the quality of life of the benign prostatic hyperplasia patients. Mod J Integrat Tradit Chin West Med 2010; 19: 4680-1.

31 Noguchi M, Kakuma T, Tomiyasu K, Kurita Y, Kukihara $\mathrm{H}$ et al. Effect of an extract of Ganoderma lucidum in men with lower urinary tract symptoms: a double-blind, placebo-controlled randomized and dose-ranging study. Asian J Androl 2008; 10: 651-8.

32 Noguchi M, Kakuma T, Tomiyasu K, Yamada A, Itoh K et al. Randomized clinical trial of an ethanol extract of Ganoderma lucidum in men with lower urinary tract symptoms. Asian J Androl 2008; 10: 777-85.

33 Zegarra L, Vaisberg A, Loza C, Aguirre RL, Campos M et al. Double-blind randomized placebo-controlled study of Bixa orellana in patients with lower urinary tract symptoms associated to benign prostatic hyperplasia. Int Braz J Urol 2007; 33: 493-500; discussion 501

34 Wen JY, Lu M, Zhou WZ, Wei XD, Wang Y et al. Treatment of 20 cases of benign prostatic hyperplasia by Tong Qiao Jian. Chin Med Shan Xi 2006; 27: 290-1.

35 Zhang FR. Clinical trial of Li Zhi soft capsule for benign prostatic hyerplasia. Chin J Clin Pharmacol Ther 2004; 9: 1424-7.
36 Zhang YD, Lu ZJ, Zhang P, Huang SG. 30 cases observation of the efficacy of Yishen zhuyu decoction for benign prostatic hyperplasia treatment. J Nanjing Tradit Chin Med Univ (Nat Sci) 2001; 17: 216-7

37 China, M.o.H.o. Clinical Research Guidelines of New Chinese Herbal Medicine. Beijing: Ministry of Health of the People's Republic of China.

38 Sun ZX, Ni LY, Huang J. Observation of the efficacy of the adjuvant use of Chinese medicine and Tamsulosin for the treatment of benign prostatic hyperplasia and related lower urinary tract symptoms. Shandong J Tradit Chin Med 2009; 28: 22-3.

39 Li S, Zhang ZQ, Wu LJ, Zhang XG, Li YD et al. Understanding ZHENG in traditional Chinese medicine in the context of neuro-endocrine-immune network. let Syst Biol 2007; 1: 51-60.

40 Benign prostatic hyperplasia. In: Li YQ, Chen HF, Cui XJ, Ai RD, He QH et al, editors. Surgery of Chinese Medicine. Beijing: Chinese Medicine Press. p293-6.

41 Yang X, Ding CF, Ni WD, Yang ZZ. Clinical study of Long shu tong for benign prostatic hyperplasia treatment. Jiangxi J Tradit Chin Med 2009; 40: 28-9.

42 Yuan ZZ. Study of efficacy and mechanism of JinGuiShenQiWan on BPH rats. In Internal Medicine of Chinese Medicine (Andrology). Chengdu: Chengdu University of Chinese Medicine; 2002; 12: 7-8.

43 Wei G. Review of the pharmacological and clinical studies of Huangqi. Shanxi J Tradit Chin Med 2011; 27: 52-4.

44 Hua ZH. Effects of Radix Astragali on blood glucose concentration and rheology of rat with diabetes mellitus. J Shantou Univ Med Coll 2005; 18: 96-8.

45 Kang HT. Book of Ingredient and Pharmacology of Common Chinese Medicine. Beijing: China Medical Science Press; 1994.

46 Liang SQ. Experimental study on the antithrombotic effect of Ehong injection. China J Chin Mater Med 1988; 15: 48

47 Lao AN, Min SS. In:Basic Research of Common Chinese Medicine. Beijing: Frontiers in China; 2002. p80-112.

48 Zang ZP, Jun WZ. Study of the pharmacology and clinical application of Cortex Phellodendri. Gansu Med J 2010; 29: 329-31. 